\title{
Right ventricular T1 mapping is technically feasible and correlates with right ventricular dysfunction in non-ischemic cardiomyopathy
}

Christine Jellis $^{1 *}$, Teerapat Yingchoncharoen ${ }^{1}$, Ayman Ayache ${ }^{1}$, Scott Flamm ${ }^{1,2}$, Deborah Kwon ${ }^{1,2}$

From 17th Annual SCMR Scientific Sessions

New Orleans, LA, USA. 16-19 January 2014

\section{Background}

T1 mapping is increasingly employed for evaluation of diffuse fibrosis within the left ventricular (LV) myocardium. Physiological confounders require correction of raw data prior to analysis. T1 values have been inversely correlated with LV systolic and diastolic function. We sought to evaluate the feasibility of right ventricular (RV) T1 mapping and to assess its relationship with RV function.

\section{Methods}

Post-contrast, T1 mapping was performed with a LookLocker technique using inversion recovery imaging in 102 consecutive subjects with non-ischemic cardiomyopathy. Regional T1 values of the RV free wall, RV septum and lateral LV were calculated using prototype analysis software. CMR volumetric data was measured offline using a cine short axis stack to establish RV end-diastolic-volume (RVEDV), end-systolic-volume (RVESV) and ejection fraction (RVEF). Simultaneous subject biochemical and anthropometric data were recorded.

\section{Results}

Subjects demonstrated mild to moderately impaired global RV systolic function (RVEF $=37 \pm 11 \%$; RVEDV =
$212 \pm 74 \mathrm{ml}$; RVESV $=140 \pm 73 \mathrm{ml})$. LV function was moderately reduced (LVEF $31 \pm 17 \%$ ). Good correlation was observed between mean LV and RV T1 values [Table 1], with similar $\mathrm{T} 1$ values maintained in both the RV free wall and septum $(r=0.601, p<0.001)$. RV free wall $\mathrm{T} 1$ values demonstrated an expected correlation with heart rate $(H R)(r=-0.253, p=0.010)$ but not other known covariates of LV T1 values (renal function, weight or contrast factors). Upon stratification according to RV free wall T1 value (Group $1 \mathrm{~T} 1<310 \mathrm{~ms}$; Group $2 \mathrm{~T} 1>310 \mathrm{~ms}$ ), RVEF was proportionally related to T1 (Group 1 mean RVEF $30 \pm 10 \%$, Group 2 mean RVEF $39 \pm 10 \% ; \mathrm{p}=0.011$ ).

\section{Conclusions}

Right ventricular T1 mapping appears technically feasible with good agreement between regional RV and LV T1 values. RV T1 values appear proportionally related to RV ejection fraction, suggestive that RV dysfunction in this population may be mediated by fibrotic factors.

\section{Funding}

No funding disclosures.

Table 1

\begin{tabular}{|c|c|c|c|c|}
\hline \multirow[t]{2}{*}{$\begin{array}{l}\text { Correlation between } \mathrm{LV} \text { and } \mathrm{RV} \text { T1 values } \\
\text { (mean T1 value) }\end{array}$} & \multicolumn{2}{|c|}{$\begin{array}{l}\text { RV free wall T1 value } \\
(350 \pm 66 \mathrm{~ms})\end{array}$} & \multicolumn{2}{|c|}{$\begin{array}{l}\text { RV septal T1 value } \\
(359 \pm 74 \mathrm{~ms})\end{array}$} \\
\hline & $r$ & $p$ & $r$ & $p$ \\
\hline Lateral LV T1 value (384 \pm 78 ms) & 0.624 & $<0.001$ & 0.646 & $<0.001$ \\
\hline
\end{tabular}

${ }^{1}$ Cardiovascular Medicine, Cleveland Clinic, Cleveland, Ohio, USA

Full list of author information is available at the end of the article 


\section{Authors' details}

${ }^{1}$ Cardiovascular Medicine, Cleveland Clinic, Cleveland, Ohio, USA. ${ }^{2}$ Diagnostic

Radiology, Cleveland Clinic, Cleveland, Ohio, USA.

Published: 16 January 2014

doi:10.1186/1532-429X-16-S1-P336

Cite this article as: Jellis et al:: Right ventricular T1 mapping is

technically feasible and correlates with right ventricular dysfunction in

non-ischemic cardiomyopathy. Journal of Cardiovascular Magnetic

Resonance 2014 16(Suppl 1):P336.

Submit your next manuscript to BioMed Central and take full advantage of:

- Convenient online submission

- Thorough peer review

- No space constraints or color figure charges

- Immediate publication on acceptance

- Inclusion in PubMed, CAS, Scopus and Google Scholar

- Research which is freely available for redistribution

Submit your manuscript at 